\title{
CHARACTERIZATION OF ALMOST SEMI-HEYTING ALGEBRA
}

\author{
V.V.V.S.S.P.S. SRIKANTH \\ M.V. RATNAMANi \\ Department of Basic Science and Humanities \\ Aditya Institute of Technology and Management \\ Tekkali, India \\ e-mail: srikanth.vvvs@gmail.com \\ vvratnamani@gmail.com \\ AND \\ S. RAMESH \\ Department of Mathematics \\ Institute of Science, GITAM (Deemed to be University) \\ Visakhapatnam, India \\ e-mail: ramesh.sirisetti@gmail.com
}

\begin{abstract}
In this paper, we initiate the discourse on the properties that hold in an almost semi-Heyting algebra but not in an semi-Heyting almost distributive lattice. We establish an equivalent condition for an almost semi-Heyting algebra to become a Stone almost distributive lattice. Moreover a glance about dense elements in an almost semi-Heyting algebra followed by study of some algebraic properties on them. Finally, we perceive that the kernel of homomorphism is equal to the dense element set.
\end{abstract}

Keywords: almost distributive lattice, semi-Heyting almost distributive lattice, almost semi-Heyting algebra, dense element and stone almost distributive lattice.

2010 Mathematics Subject Classification: Primary 06D99; Secondary $06 \mathrm{D} 20$. 


\section{REFERENCES}

[1] R.F. Arens and I. Kaplansky, The topological representation of algebras, Trans. Amer. Math. Soc. 63 (1948) 457-481. doi:10.1090/S0002-9947-1948-0025453-6

[2] G. Birkhoff, Lattice Theory, 3rd ed., Colloq. Publ. Amer. Math. Soc. 25 (Providence, R.I., 1979).

[3] N.H. McCoy and D. Mantgomery, A representation of generalized Boolean ring, Duke. Math. 3 (1937) 455-459. doi:10.1215/S0012-7094-37-00335-1

[4] G.C. Rao, M.V. Ratnamani, K.P. Shum and Berhanu Assaye, Semi-Heyting almost distributive lattices, Lobachevskii J. Math. 36 (2015) 184-189. doi:10.1134/S1995080215020158

[5] G.C. Rao, M.V. Ratnamani and K.P. Shum, Almost semi-Heyting algebra SEA Bull. Math. 42 (2018) 95-110.

[6] A. Horn, Logic with truth values in linerly ordered Heyting algebrra, J. Symb. Logics 34 (1969) 395-408. doi: $10.2307 / 2270905$

[7] H.P. Sankappnaver, Dually quasi-De Morgan Stone semi-Heyting algebras I. Regularity, Category and General Algebraic Structures with Applications 2 (2014) 47-64.

[8] H.P. Sankappnaver, Expansion of semi-Heyting algebra, Studia Logica 98 (2011) $27-81$. doi:10.1007/S11225-011-9322-6

[9] H.P. Sankppnaver, Heyting algebras with dual pseudocomplementation, Pacific J. Math. 107 (1985) 405-415. doi:10.2140/pjm.1985.117.405

[10] H.P. Sankappanavar, Semi-Heyting algebra: an abstraction from Heyting algebras, IX Congreso Monteiro (2007) 33-66.

[11] T. Skolem, Logico-combinatorial Investigations in the Satisfiability or Provability of Mathematical Propositions (Harvard University Press, Cambridge, 1920).

[12] N.V. Subrahmanyam, Lattice theory for certain classes of rings, Math. Ann. 139 (1960) 275-286. doi:10.1007/BF01352263

[13] N.V. Subrahmanyam, An extension of Boolean lattice theory, Math. Ann. 151 (1963) 332-345. doi:10.1007/BF01470824

[14] I. Sussman, A generalization for Boolean rings, Math. Ann. Bd. 136 (1958) 326-338. doi:10.1007/BF01360238

[15] U.M. Swamy and G.C. Rao, Almost distributive lattices, J. Asust. Math. Soc. 31 (1981) 77-91. doi:10.1017/S1446788700018498 
[16] U.M. Swamy and G.C. Rao, Stone almost distributive lattices, SEA Bull. Math. 27 (2003) 513-526.

[17] U.M. Swamy, G.C. Rao and G. Rao Nanaji, Pseudo-complementation on almost distributive lattices, SEA Bull. Math. 24 (2000) 95-104.

[18] J. Von Neumann, On regular rings, Proc. Nat. Acad. Sci. U.S.A. 22 (1936) 707-713.

Received 10 October 2019

Revised 11 February 2020

Accepted 26 June 20202 